PROCEEDINGS OF THE

AMERICAN MATHEMATICAL SOCIETY

Volume 132, Number 11, Pages 3441-3448

S 0002-9939(04)07480-5

Article electronically published on May 12, 2004

\title{
DENSE SUBSETS OF THE BOUNDARY OF A COXETER SYSTEM
}

\author{
TETSUYA HOSAKA
}

(Communicated by Ronald A. Fintushel)

\begin{abstract}
In this paper, we investigate dense subsets of the boundary of a Coxeter system. We show that for a Coxeter system $(W, S)$, if $W^{\left\{s_{0}\right\}}$ is quasi-dense in $W$ and the order $o\left(s_{0} t_{0}\right)=\infty$ for some $s_{0}, t_{0} \in S$, then there exists a point $\alpha$ in the boundary $\partial \Sigma(W, S)$ of the Coxeter system $(W, S)$ such that the orbit $W \alpha$ is dense in $\partial \Sigma(W, S)$. Here $W^{\left\{s_{0}\right\}}=\{w \in W \mid \ell(w s)<$ $\ell(w)$ for each $\left.s \in S \backslash\left\{s_{0}\right\}\right\} \backslash\{1\}$. We also show that if the set $\bigcup\left\{W^{\{s\}} \mid s \in\right.$ $S$ such that $o(s t)=\infty$ for some $t \in S\}$ is quasi-dense in $W$, then $\left\{w^{\infty} \mid w \in\right.$ $W$ such that $o(w)=\infty\}$ is dense in $\partial \Sigma(W, S)$.
\end{abstract}

\section{INTRODUCTION AND PRELIMINARIES}

The purpose of this paper is to study dense subsets of the boundary of a Coxeter system. A Coxeter group is a group $W$ having a presentation

$$
\left.\langle S|(s t)^{m(s, t)}=1 \text { for } s, t \in S\right\rangle,
$$

where $S$ is a finite set and $m: S \times S \rightarrow \mathbb{N} \cup\{\infty\}$ is a function satisfying the following conditions:

(1) $m(s, t)=m(t, s)$ for each $s, t \in S$,

(2) $m(s, s)=1$ for each $s \in S$, and

(3) $m(s, t) \geq 2$ for each $s, t \in S$ such that $s \neq t$.

The pair $(W, S)$ is called a Coxeter system. Let $(W, S)$ be a Coxeter system. For a subset $T \subset S, W_{T}$ is defined as the subgroup of $W$ generated by $T$, and called a parabolic subgroup. If $T$ is the empty set, then $W_{T}$ is the trivial group. A subset $T \subset S$ is called a spherical subset of $S$, if the parabolic subgroup $W_{T}$ is finite. For each $w \in W$, we define $S(w)=\{s \in S \mid \ell(w s)<\ell(w)\}$, where $\ell(w)$ is the minimum length of a word in $S$ which represents $w$. For a subset $T \subset S$, we also define $W^{T}=\{w \in W \mid S(w)=T\}$.

Let $(W, S)$ be a Coxeter system and let $\mathcal{S}^{f}$ be the family of spherical subsets of $S$. We denote by $W \mathcal{S}^{f}$ the set of all cosets of the form $w W_{T}$, with $w \in W$ and $T \in \mathcal{S}^{f}$. The sets $\mathcal{S}^{f}$ and $W \mathcal{S}^{f}$ are partially ordered by inclusion. Contractible simplicial complexes $K(W, S)$ and $\Sigma(W, S)$ are defined as the geometric realizations

Received by the editors April 15, 2003 and, in revised form, August 4, 2003.

2000 Mathematics Subject Classification. Primary 57M07, 20F65, 20 F55.

Key words and phrases. Boundaries of Coxeter groups.

The author was partly supported by a Grant-in-Aid for Scientific Research, The Ministry of Education, Culture, Sports, Science and Technology, Japan, (No. 15740029).

(C)2004 American Mathematical Society 
of the partially ordered sets $\mathcal{S}^{f}$ and $W \mathcal{S}^{f}$, respectively ([7, §3], [5]). The natural embedding $\mathcal{S}^{f} \rightarrow W \mathcal{S}^{f}$ defined by $T \mapsto W_{T}$ induces an embedding $K(W, S) \rightarrow$ $\Sigma(W, S)$ which we regard as an inclusion. The group $W$ acts on $\Sigma(W, S)$ via a simplicial automorphism. Then $\Sigma(W, S)=W K(W, S)$ and $\Sigma(W, S) / W \cong K(W, S)$ ([5], 7]). For each $w \in W, w K(W, S)$ is called a chamber of $\Sigma(W, S)$. If $W$ is infinite, then $\Sigma(W, S)$ is noncompact. In 11], G. Moussong proved that a natural metric on $\Sigma(W, S)$ satisfies the $\operatorname{CAT}(0)$ condition. Hence, if $W$ is infinite, $\Sigma(W, S)$ can be compactified by adding its ideal boundary $\partial \Sigma(W, S)([6, \S 4]$, [8]). This boundary $\partial \Sigma(W, S)$ is called the boundary of $(W, S)$. We note that the natural action of $W$ on $\Sigma(W, S)$ is properly discontinuous and cocompact ([5], [6]), and this action induces an action of $W$ on $\partial \Sigma(W, S)$.

A subset $A$ of a space $X$ is said to be dense in $X$, if $\bar{A}=X$. A subset $A$ of a metric space $X$ is said to be quasi-dense, if there exists $N>0$ such that each point of $X$ is $N$-close to some point of $A$.

Let $(W, S)$ be a Coxeter system. Then $W$ has the word metric $d_{\ell}$ defined by $d_{\ell}\left(w, w^{\prime}\right)=\ell\left(w^{-1} w^{\prime}\right)$ for any $w, w^{\prime} \in W$.

After some preliminaries in Sections 2 and 3, we prove the following theorems in Section 4 .

Theorem 1. Let $(W, S)$ be a Coxeter system. Suppose that $W^{\left\{s_{0}\right\}}$ is quasi-dense in $W$ with respect to the word metric and $o\left(s_{0} t_{0}\right)=\infty$ for some $s_{0}, t_{0} \in S$, where $o\left(s_{0} t_{0}\right)$ is the order of $s_{0} t_{0}$ in $W$. Then there exists $\alpha \in \partial \Sigma(W, S)$ such that the orbit $W \alpha$ is dense in $\partial \Sigma(W, S)$.

Suppose that a group $\Gamma$ acts properly and cocompactly by isometries on a $\operatorname{CAT}(0)$ space $X$. Every element $\gamma \in \Gamma$ such that $o(\gamma)=\infty$ is a hyperbolic transformation of $X$, i.e., there exist a geodesic axis $c: \mathbb{R} \rightarrow X$ and a real number $a>0$ such that $\gamma \cdot c(t)=c(t+a)$ for each $t \in \mathbb{R}(\underline{3})$. Then, for all $x \in X$, the sequence $\left\{\gamma^{i} x\right\}$ converges to $c(\infty)$ in $X \cup \partial X$. We denote $\gamma^{\infty}=c(\infty)$.

Theorem 2. Let $(W, S)$ be a Coxeter system. If the set

$$
\bigcup\left\{W^{\{s\}} \mid s \in S \text { such that } o(s t)=\infty \text { for some } t \in S\right\}
$$

is quasi-dense in $W$, then $\left\{w^{\infty} \mid w \in W\right.$ such that $\left.o(w)=\infty\right\}$ is dense in $\partial \Sigma(W, S)$.

Remark. For a negatively curved group $G$ and the boundary $\partial G$ of $G$,

(1) we can show that $G \alpha$ is dense in $\partial G$ for each $\alpha \in \partial G$ by an easy argument, and

(2) it is known that $\left\{g^{\infty} \mid g \in G\right.$ such that $\left.o(g)=\infty\right\}$ is dense in $\partial G$ ([2]).

Example. Let $S=\{s, t, u\}$ and let

$$
W=\left\langle S \mid s^{2}=t^{2}=u^{2}=(s t)^{3}=(t u)^{3}=(u s)^{3}=1\right\rangle .
$$

Then $(W, S)$ is a Coxeter system and $W^{\{s\}}$ is quasi-dense in $W$. On the other hand, for any $\alpha \in \partial \Sigma(W, S), W \alpha$ is a finite-points set and not dense in $\partial \Sigma(W, S)$, which is a circle. Thus we cannot omit the assumption " $o\left(s_{0} t_{0}\right)=\infty$ " in Theorem 1

As an application of Theorems 1 and 2] we show the following corollary.

Corollary 3. Let $(W, S)$ be a Coxeter system. Suppose that there exist a maximal spherical subset $T$ of $S$ and an element $s_{0} \in S$ such that $o\left(s_{0} t\right) \geq 3$ for each $t \in T$ 
and $o\left(s_{0} t_{0}\right)=\infty$ for some $t_{0} \in T$. Then

(1) $W \alpha$ is dense in $\partial \Sigma(W, S)$ for some $\alpha \in \partial \Sigma(W, S)$, and

(2) $\left\{w^{\infty} \mid w \in W\right.$ such that $\left.o(w)=\infty\right\}$ is dense in $\partial \Sigma(W, S)$.

Example. The Coxeter system defined by the diagram in Figure 1 is not hyperbolic in Gromov's sense, since it contains a copy of $\mathbb{Z}^{2}$, and it satisfies the condition of Corollary 3

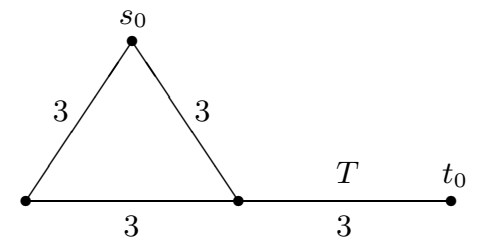

FiguRe 1.

\section{Lemmas on COXeter groups}

In this section, we prove some lemmas for Coxeter groups which are used later.

Definition 2.1. Let $(W, S)$ be a Coxeter system and $w \in W$. A representation $w=s_{1} \cdots s_{l}\left(s_{i} \in S\right)$ is said to be reduced, if $\ell(w)=l$, where $\ell(w)$ is the minimum length of a word in $S$ which represents $w$.

The following lemma is known.

Lemma 2.2 ([1], 4], [5, 7], [10]). Let $(W, S)$ be a Coxeter system.

(1) Let $w \in W$ and let $w=s_{1} \cdots s_{l}$ be a representation. If $\ell(w)<l$, then $w=s_{1} \cdots \hat{s_{i}} \cdots \hat{s_{j}} \cdots s_{l}$ for some $1 \leq i<j \leq l$.

(2) For each $w \in W$ and $s \in S$, $\ell(w s)$ equals either $\ell(w)+1$ or $\ell(w)-1$, and $\ell(s w)$ also equals either $\ell(w)+1$ or $\ell(w)-1$.

(3) For each $w \in W, S(w)$ is a spherical subset of $S$, i.e., $W_{S(w)}$ is finite.

(4) For each $w \in W$ and $u \in W_{S \backslash S(w)}, \ell(w u)=\ell(w)+\ell(u)$.

(5) For each $w \in W$ and $u \in W_{S(w)}, \ell(w u)=\ell(w)-\ell(u)$, i.e., if $u=t_{1} \cdots t_{k}$ $\left(t_{i} \in S\right)$ is a reduced representation, then there exists a reduced representation $w=s_{1} \cdots s_{l}\left(s_{j} \in S\right)$ such that $t_{i}=s_{l-k+i}$ for each $i=1, \ldots, k$.

Lemma 2.3. Let $(W, S)$ be a Coxeter system, $w \in W$ and $s, t \in S$. Suppose that $\ell(w s)=\ell(w)+1$ and $s \neq t$.

(1) If $o(s t)=\infty$, then $\ell(w s t)=\ell(w)+2$.

(2) If $\ell(w s t)=\ell(w)$, then $t \in S(w)$.

(3) If o(st) $=\infty$, then $W^{\{s\}} t \subset W^{\{t\}}$.

Proof. (1) By Lemma2.2 (2), either $\ell(w s t)=\ell(w)$ or $\ell(w s t)=\ell(w)+2$. We suppose that $\ell(w s t)=\ell(w)$. Then $\{s, t\} \subset S(w s)$, and $W_{\{s, t\}}$ is finite by Lemma 2.2 (3). This means that $o(s t)<\infty$. Hence if $o(s t)=\infty$, then $\ell(w s t)=\ell(w)+2$.

(2) Suppose that $t \notin S(w)$. Since $\{s, t\} \subset S \backslash S(w)$, it follows that $\ell(w s t)=$ $\ell(w)+\ell(s t)=\ell(w)+2$ by Lemma $2.2(4)$. Thus if $\ell(w s t)=\ell(w)$, then $t \in S(w)$.

(3) Suppose that $o(s t)=\infty$. Let $w \in W^{\{s\}}$. Since $t \notin S(w)=\{s\}$, it follows that $\ell(w t)=\ell(w)+1$. Let $u \in S \backslash\{t\}$. If $u=s$, then $\ell(w t u)=\ell(w)+2=\ell(w t)+1$ 
by $(1)$, because $o(s t)=\infty$. If $u \neq s$, then $u \notin S(w)=\{s\}$ and $\ell(w t u)=\ell(w t)+1$ by (2). Hence $\ell(w t u)=\ell(w t)+1$ for each $u \in S \backslash\{t\}$; that is, $w t \in W^{\{t\}}$. Thus $W^{\{s\}} t \subset W^{\{t\}}$.

We prove the following lemmas concerning the set $W^{\{s\}}$.

Lemma 2.4. Let $(W, S)$ be a Coxeter system, $w \in W$ and $s_{0} \in S$. Suppose that $o\left(s_{0} t\right) \geq 3$ for each $t \in S(w)$, and that $o\left(s_{0} t_{0}\right)=\infty$ for some $t_{0} \in S(w)$. Then $w s_{0} \in W^{\left\{s_{0}\right\}}$.

Proof. Suppose that $o\left(s_{0} t\right) \geq 3$ for each $t \in S(w)$, and that $o\left(s_{0} t_{0}\right)=\infty$ for some $t_{0} \in S(w)$. To prove that $w s_{0} \in W^{\left\{s_{0}\right\}}$, we show that $\ell\left(w s_{0} t\right)=\ell\left(w s_{0}\right)+1$ for each $t \in S \backslash\left\{s_{0}\right\}$. Let $t \in S \backslash\left\{s_{0}\right\}$. If $t=t_{0}$, then $\ell\left(w s_{0} t\right)=\ell\left(w s_{0}\right)+1$ by Lemma 2.3 (1), since $o\left(s_{0} t_{0}\right)=\infty$. Now we suppose that $\ell\left(w s_{0} t\right)=\ell\left(w s_{0}\right)-1$ for some $t \in S \backslash\left\{s_{0}, t_{0}\right\}$. Then $t \in S(w)$ by Lemma 2.3 (2). Hence $o\left(s_{0} t\right) \geq 3$ by hypothesis, and $s_{0} t s_{0}$ is reduced. Since $\left\{s_{0}, t\right\} \subset S\left(w s_{0}\right)$, there exists a reduced representation $w s_{0}=\left(a_{1} \cdots a_{l-2}\right) s_{0} t s_{0}$ by Lemma $2.2(5)$, where $l=\ell(w)$. Then $w t=\left(a_{1} \cdots a_{l-2}\right) s_{0}$ is reduced. On the other hand, since $\left\{t, t_{0}\right\} \subset S(w)$, there exists a reduced representation $w=\left(a_{1}^{\prime} \cdots a_{l-2}^{\prime}\right) t_{0} t$. Hence $w t=\left(a_{1}^{\prime} \cdots a_{l-2}^{\prime}\right) t_{0}$ is reduced. Thus we have two reduced representations

$$
\left(a_{1} \cdots a_{l-2}\right) s_{0}=w t=\left(a_{1}^{\prime} \cdots a_{l-2}^{\prime}\right) t_{0} .
$$

This means that $\left\{s_{0}, t_{0}\right\} \subset S(w t)$, i.e., $o\left(s_{0} t_{0}\right)<\infty$ by Lemma 2.2 (3). This contradicts the assumption $o\left(s_{0} t_{0}\right)=\infty$. Hence $\ell\left(w s_{0} t\right)=\ell\left(w s_{0}\right)+1$ for each $t \in S \backslash\left\{s_{0}\right\}$; that is, $w s_{0} \in W^{\left\{s_{0}\right\}}$.

Lemma 2.5. Let $(W, S)$ be a Coxeter system. Suppose that there exist a maximal spherical subset $T$ of $S$ and $s_{0} \in S$ such that $o\left(s_{0} t\right) \geq 3$ for each $t \in T$ and $o\left(s_{0} t_{0}\right)=\infty$ for some $t_{0} \in T$. Then $W^{\left\{s_{0}\right\}}$ is quasi-dense in $W$.

Proof. Let $w \in W$. Since $T$ is a spherical subset of $S$, there exists an element $w^{\prime}$ of longest length in the coset $w W_{T}$. Then we show that $S\left(w^{\prime}\right)=T$.

Let $t \in T$. Since $w^{\prime} t \in w^{\prime} W_{T}=w W_{T}$ and $w^{\prime}$ is the element of longest length in $w W_{T}$, it follows that $\ell\left(w^{\prime} t\right)<\ell\left(w^{\prime}\right)$, i.e., $t \in S\left(w^{\prime}\right)$. Thus $T \subset S\left(w^{\prime}\right)$. Now $T$ is a maximal spherical subset of $S$, and $S\left(w^{\prime}\right)$ is a spherical subset of $S$ by Lemma 2.2 (3). Hence $S\left(w^{\prime}\right)=T$.

Then $w^{\prime} s_{0} \in W^{\left\{s_{0}\right\}}$ by hypothesis and Lemma 2.4. Here

$$
d_{\ell}\left(w, w^{\prime} s_{0}\right)=\ell\left(w^{-1} w^{\prime} s_{0}\right) \leq \max \left\{\ell(v) \mid v \in W_{T}\right\}+1,
$$

because $w^{-1} w^{\prime} \in W_{T}$. Thus $W^{\left\{s_{0}\right\}}$ is quasi-dense in $W$.

\section{A CAT(0) Space on Which a Coxeter group aCts}

In this section, we give some lemmas for the $\operatorname{CAT}(0)$ space $\Sigma(W, S)$.

We first recall some basic properties of $\operatorname{CAT}(0)$ spaces. Details of $\mathrm{CAT}(0)$ spaces and their boundaries are found in 3 and $[8$. We say that a metric space $(X, d)$ is a geodesic space if for each $x, y \in X$, there exists an isometry $\xi:[0, d(x, y)] \rightarrow X$ such that $\xi(0)=x$ and $\xi(d(x, y))=y$ (such a $\xi$ is called a geodesic). Also a metric space $(X, d)$ is said to be proper if every closed metric ball is compact.

Let $(X, d)$ be a geodesic space. Two geodesic rays $\xi, \zeta:[0, \infty) \rightarrow X$ are said to be asymptotic if there exists a constant $N$ such that $d(\xi(t), \zeta(t)) \leq N$ for each $t \geq 0$. 
The following proposition is known.

Proposition 3.1 (cf. [3], 8]). Let $(X, d)$ be a proper $C A T(0)$ space.

(1) For any two points $x, y \in X$, there exists a unique geodesic segment between $x$ and $y$ in $X$.

(2) $X$ is contractible.

(3) For each geodesic ray $\xi$ in $X$ and each point $x_{0} \in X$, there exists a unique geodesic ray $\xi^{\prime}$ issuing from $x_{0}$ such that $\xi$ and $\xi^{\prime}$ are asymptotic.

(4) For any three points $x_{0}, x_{1}, x_{2} \in X$ and each $t \in[0,1]$,

$$
d\left(\xi_{1}\left(t d\left(x_{0}, x_{1}\right)\right), \xi_{2}\left(t d\left(x_{0}, x_{2}\right)\right)\right) \leq t d\left(x_{1}, x_{2}\right),
$$

where $\xi_{i}:\left[0, d_{i}\right] \rightarrow X$ is the geodesic segment from $x_{0}$ to $x_{i}$ for each $i=1,2$.

Let $(X, d)$ be a proper $\mathrm{CAT}(0)$ space and $x_{0} \in X$. The boundary of $X$ with respect to $x_{0}$, denoted by $\partial_{x_{0}} X$, is defined as the set of all geodesic rays issuing from $x_{0}$. Then $X \cup \partial_{x_{0}} X$ has a natural topology, in which $X$ is an open subspace, and a neighborhood basis for each point $\xi \in \partial_{x_{0}} X$ is given by the sets

$$
U(\xi ; r, \epsilon)=\left\{x \in X \cup \partial X \mid x \notin B\left(x_{0}, r\right), d\left(\xi(r), \xi_{x}(r)\right)<\epsilon\right\},
$$

where $r, \epsilon>0$ and $\xi_{x}:\left[0, d\left(x_{0}, x\right)\right] \rightarrow X$ is the geodesic from $x_{0}$ to $x\left(\xi_{x}=x\right.$ if $\left.x \in \partial_{x_{0}} X\right)$. This is called the cone topology on $X \cup \partial_{x_{0}} X$. It is known that $X \cup \partial_{x_{0}} X$ is a metrizable compactification of $X([\underline{3}, \underline{8}])$.

Let $x_{0}$ and $x_{1}$ be two points of a proper CAT(0) space $X$. By Proposition $3.1(3)$, there exists a unique bijection $\Phi: \partial_{x_{0}} X \rightarrow \partial_{x_{1}} X$ such that $\xi$ and $\Phi(\xi)$ are asymptotic for each $\xi \in \partial_{x_{0}} X$. It is known that $\Phi: \partial_{x_{0}} X \rightarrow \partial_{x_{1}} X$ is a homeomorphism $(3], 8]$.

Let $X$ be a proper $\mathrm{CAT}(0)$ space. The asymptotic relation is an equivalence relation in the set of all geodesic rays in $X$. The boundary of $X$, denoted by $\partial X$, is defined as the set of asymptotic equivalence classes of geodesic rays. The equivalence class of a geodesic ray $\xi$ is denoted by $\xi(\infty)$. By Proposition [3.1 (3), for each $x_{0} \in X$ and each $\alpha \in \partial X$, there exists a unique element $\xi \in \partial_{x_{0}} X$ with $\xi(\infty)=\alpha$. Thus we may identify $\partial X$ with $\partial_{x_{0}} X$ for each $x_{0} \in X$.

Let $(X, d)$ be a proper $\operatorname{CAT}(0)$ space and $\Gamma$ a group which acts on $X$ by isometries. For each element $\gamma \in \Gamma$ and each geodesic ray $\xi:[0, \infty) \rightarrow X$, a map $\gamma \xi:[0, \infty) \rightarrow X$ defined by $(\gamma \xi)(t):=\gamma(\xi(t))$ is also a geodesic ray. If geodesic rays $\xi$ and $\xi^{\prime}$ are asymptotic, then $\gamma \xi$ and $\gamma \xi^{\prime}$ are also asymptotic. Thus $\gamma$ induces a homeomorphism of $\partial X$ and $\Gamma$ acts on $\partial X$.

Let $(W, S)$ be a Coxeter system and let $\Sigma(W, S)$ and $K(W, S)$ be the proper CAT $(0)$ cell complex and its chamber defined in Section 1, respectively. Here $\Sigma(W, S)$ has two structures. The definition of $\Sigma(W, S)$ in Section 1 coincides with the barycentric subdivision of the Moussong cellular decomposition, which can be found in [1] and [6]. Now we consider the Moussong cellular decomposition. Then each $n$-cell of $\Sigma(W, S)$ is a convex subspace of the $n$-dimensional Euclidean space, and the vertex set of each cell of $\Sigma(W, S)$ is the form $w W_{T}$ with $w \in W$ and $T \in \mathcal{S}^{f}$ ([1], 6]). We note that the vertex set of $\Sigma(W, S)$ is $W$, and the 1 -skeleton $\Sigma(W, S)^{(1)}$ is the Cayley graph of $W$ with respect to $S$ with unit edges. For each subset $T \subset S, \Sigma\left(W_{T}, T\right)$ is a subcomplex of $\Sigma(W, S)$.

For each representation $w=s_{1} \cdots s_{l} \in W$, we consider the path $P_{s_{1}, \ldots, s_{l}}:=\left[1, s_{1}\right] \cup\left[s_{1},\left(s_{1} s_{2}\right)\right] \cup \cdots \cup\left[\left(s_{1} \cdots s_{l-2}\right),\left(s_{1} \cdots s_{l-1}\right)\right] \cup\left[\left(s_{1} \cdots s_{l-1}\right), w\right]$ in $\Sigma(W, S)$. 
The following lemma was proved in 9 .

Lemma 3.2 ([9, Lemma 4.2]). Let $(W, S)$ be a Coxeter system and $N$ the diameter of $K(W, S)$ in $\Sigma(W, S)$. Then for each $(1 \neq) w \in W$, there exists a reduced representation $w=s_{1} \cdots s_{l}$ such that

$$
d_{H}\left(\operatorname{Im} \xi_{w}, P_{s_{1}, \ldots, s_{l}}\right) \leq N
$$

where $d_{H}$ is the Hausdorff distance and $\xi_{w}$ is the geodesic from 1 to $w$ in $\Sigma(W, S)$.

Using this lemma and a consequence of Tits' solution to the word problem ([12], [4, p.50]), we show the following lemma.

Lemma 3.3. Let $(W, S)$ be a Coxeter system and $x, y \in W$. If $o(s t)=\infty$ for each $s \in S(x)$ and $t \in S\left(y^{-1}\right)$, then $d\left(x, \operatorname{Im} \xi_{x y}\right) \leq N$, where $\xi_{x y}$ is the geodesic from 1 to $x y$ in $\Sigma(W, S)$ and $N$ is the diameter of $K(W, S)$ in $\Sigma(W, S)$.

Proof. Suppose that $o(s t)=\infty$ for each $s \in S(x)$ and $t \in S\left(y^{-1}\right)$. Let $x=s_{1} \cdots s_{l}$ and $y=t_{1} \cdots t_{k}$ be reduced representations. Then

$$
x y=\left(s_{1} \cdots s_{l}\right)\left(t_{1} \cdots t_{k}\right)
$$

is reduced. By Lemma [3.2, there exists a reduced representation $x y=a_{1} \cdots a_{l+k}$ such that

$$
d_{H}\left(\operatorname{Im} \xi_{x y}, P_{a_{1}, \ldots, a_{l+k}}\right) \leq N .
$$

Since $o(s t)=\infty$ for each $s \in S(x)$ and $t \in S\left(y^{-1}\right)$, it follows that $a_{1} \cdots a_{l}=x$ by Tits's theorem in [12] and [4, p.50]. Hence $x \in P_{a_{1}, \ldots, a_{l+k}}$. Thus $d\left(x, \operatorname{Im} \xi_{x y}\right) \leq$ $N$.

\section{Proof of the main Results}

Using some lemmas in Sections 2 and 3, we prove the main results.

Theorem 4.1. Let $(W, S)$ be a Coxeter system. If $W^{\left\{s_{0}\right\}}$ is quasi-dense in $W$ and $o\left(s_{0} t_{0}\right)=\infty$ for some $s_{0}, t_{0} \in S$, then there exists an $\alpha \in \partial \Sigma(W, S)$ such that $W \alpha$ is dense in $\partial \Sigma(W, S)$.

Proof. Suppose that for some $s_{0}, t_{0} \in S, W^{\left\{s_{0}\right\}}$ is quasi-dense in $W$ and $o\left(s_{0} t_{0}\right)=$ $\infty$. Then $W^{\left\{s_{0}\right\}} t_{0} \subset W^{\left\{t_{0}\right\}}$ by Lemma 2.3 (3). Hence $W^{\left\{t_{0}\right\}}$ is infinite, and there exists a sequence $\left\{x_{j}\right\} \subset\left(W^{\left\{t_{0}\right\}}\right)^{-1}$ which converges to some point $\alpha \in \partial \Sigma(W, S)$ in $\Sigma(W, S) \cup \partial \Sigma(W, S)$. Then we show that $W \alpha$ is dense in $\partial \Sigma(W, S)$.

Let $\beta \in \partial \Sigma(W, S)$. Since $W^{\left\{s_{0}\right\}}$ is quasi-dense in $W$, we have $W^{\left\{s_{0}\right\}} B(1, M)=$ $\Sigma(W, S)$ for some $M>0$, where $B(x, r)$ is the metric ball of radius $r$ about $x$. Then there exists a sequence $\left\{w_{i}\right\} \subset W^{\left\{s_{0}\right\}}$ which converges to $\beta$ in $\Sigma(W, S) \cup \partial \Sigma(W, S)$ such that $d\left(w_{i}, \operatorname{Im} \xi_{\beta}\right) \leq M$ for each $i$, where $\xi_{\beta}$ is the geodesic ray issuing from 1 such that $\xi_{\beta}(\infty)=\beta$. We show that the sequence $\left\{w_{i} \alpha\right\}$ converges to $\beta$ in $\partial \Sigma(W, S)$. Now $w_{i} \in W^{\left\{s_{0}\right\}}, x_{j} \in\left(W^{\left\{t_{0}\right\}}\right)^{-1}$ and $o\left(s_{0} t_{0}\right)=\infty$. Hence $d\left(w_{i}, \operatorname{Im} \xi_{w_{i} x_{j}}\right) \leq N$ by Lemma 3.3 Here $\left\{w_{i} x_{j}\right\}_{j}$ converges to $w_{i} \alpha$, and $d\left(w_{i}, \operatorname{Im} \xi_{w_{i} \alpha}\right) \leq N$ for each $i$. We note that $d\left(w_{i}, \operatorname{Im} \xi_{\beta}\right) \leq M$ for each $i$. Thus the sequence $\left\{w_{i} \alpha\right\}$ converges to $\beta$ in $\partial \Sigma(W, S)$. This means that $\beta \in \overline{W \alpha}$. Hence $W \alpha$ is dense in $\partial \Sigma(W, S)$.

Theorem 4.2. Let $(W, S)$ be a Coxeter system. If the set

$$
\bigcup\left\{W^{\{s\}} \mid s \in S \text { such that } o(s t)=\infty \text { for some } t \in S\right\}
$$

is quasi-dense in $W$, then $\left\{w^{\infty} \mid w \in W\right.$ such that $\left.o(w)=\infty\right\}$ is dense in $\partial \Sigma(W, S)$. 
Proof. Suppose that $A:=\bigcup\left\{W^{\{s\}} \mid s \in S\right.$ such that $o(s t)=\infty$ for some $\left.t \in S\right\}$ is quasi-dense in $W$.

We first show that for each $w \in A$, there exists $x \in W$ such that $o(x)=\infty$ and $d\left(w, \operatorname{Im} \xi_{x^{\infty}}\right) \leq N$, where $N$ is the diameter of $K(W, S)$ in $\Sigma(W, S)$ and $\xi_{x^{\infty}}$ is the geodesic ray issuing from 1 such that $\xi_{x \infty}(\infty)=x^{\infty}$.

Let $w \in A$. Then $w \in W^{\left\{s_{0}\right\}}$ and $m\left(s_{0}, t_{0}\right)=\infty$ for some $s_{0}, t_{0} \in S$. Let $x=$ $w t_{0} s_{0} w^{-1}$. Then $x^{n}=w\left(t_{0} s_{0}\right)^{n} w^{-1}$ for each $n \geq 1$. We show that $d\left(w, \operatorname{Im} \xi_{x}\right) \leq$ $N$. Let

$$
R=\sup \left\{r \in[0, \infty) \mid d\left(w, \xi_{w\left(t_{0} s_{0}\right)^{n}}(r)\right) \leq N \text { for some } n \in \mathbb{N}\right\},
$$

where we note that $R$ is finite. Since $w \in W^{\left\{s_{0}\right\}}$ and $m\left(s_{0}, t_{0}\right)=\infty$, for each $n$ we have $\ell\left(w\left(t_{0} s_{0}\right)^{n}\right)=\ell(w)+2 n$ by Lemma 2.3, and $d\left(w, \operatorname{Im} \xi_{w\left(t_{0} s_{0}\right)^{n}}\right) \leq N$ by Lemma 3.3. Hence, for each $n$, there exists $r_{n} \in[0, R]$ such that

$$
d\left(w, \xi_{w\left(t_{0} s_{0}\right)^{n}}\left(r_{n}\right)\right) \leq N .
$$

Then by Proposition 3.1 (4),

$$
\begin{aligned}
d\left(\xi_{w\left(t_{0} s_{0}\right)^{n}}\left(r_{n}\right), \operatorname{Im} \xi_{x^{n}}\right) & =d\left(\xi_{w\left(t_{0} s_{0}\right)^{n}}\left(r_{n}\right), \operatorname{Im} \xi_{w\left(t_{0} s_{0}\right)^{n} w^{-1}}\right) \\
& \leq \frac{r_{n}}{d\left(1, w\left(t_{0} s_{0}\right)^{n}\right)} d\left(w\left(t_{0} s_{0}\right)^{n}, w\left(t_{0} s_{0}\right)^{n} w^{-1}\right) \\
& \leq \frac{R}{\frac{1}{\lambda}(\ell(w)+2 n)-C} \ell(w) \\
& =\frac{\lambda R \ell(w)}{\ell(w)+2 n-\lambda C},
\end{aligned}
$$

where $\lambda$ and $C$ are constants such that

$$
d_{\ell}(u, v)=\ell\left(u^{-1} v\right) \leq \lambda d(u, v)+C
$$

for any $u, v \in W$ (cf. [3 p.140]). Here

$$
\frac{\lambda R \ell(w)}{\ell(w)+2 n-\lambda C} \rightarrow 0 \text { as } n \rightarrow \infty .
$$

Thus

$$
d\left(w, \operatorname{Im} \xi_{x \infty}\right) \leq N .
$$

For each $\beta \in \partial \Sigma(W, S)$, there exists a sequence $\left\{w_{i}\right\} \subset A$ which converges to $\beta$, because $A$ is quasi-dense in $W$. By the above argument, there exists a sequence $\left\{x_{i}\right\} \subset W$ such that $o\left(x_{i}\right)=\infty$ and $d\left(w_{i}, \operatorname{Im} \xi_{x_{i}^{\infty}}\right) \leq N$ for each $i$. Then the sequence $\left\{x_{i}^{\infty}\right\}$ converges to $\beta$ in $\partial \Sigma(W, S)$, since $\left\{w_{i}\right\}$ converges to $\beta$. Therefore $\left\{w^{\infty} \mid w \in W\right.$ such that $\left.o(w)=\infty\right\}$ is dense in $\partial \Sigma(W, S)$.

We can obtain the following corollary from Lemma 2.5 and Theorems 4.1 and 4.2 .

Corollary 4.3. Let $(W, S)$ be a Coxeter system. Suppose that there exist a maximal spherical subset $T$ of $S$ and $s_{0} \in S$ such that $o\left(s_{0} t\right) \geq 3$ for each $t \in T$ and $o\left(s_{0} t_{0}\right)=\infty$ for some $t_{0} \in T$. Then

(1) $W \alpha$ is dense in $\partial \Sigma(W, S)$ for some $\alpha \in \partial \Sigma(W, S)$, and

(2) $\left\{w^{\infty} \mid w \in W\right.$ such that $\left.o(w)=\infty\right\}$ is dense in $\partial \Sigma(W, S)$. 


\section{REFERENCES}

[1] N. Bourbaki, Groupes et Algebrès de Lie, Chapters IV-VI, Masson, Paris, 1981. MR 39:1590

[2] P. L. Bowers and K. Ruane, Fixed points in boundaries of negatively curved groups, Proc. Amer. Math. Soc. 124 (1996), 1311-1313. MR 96g:20052

[3] M. R. Bridson and A. Haefliger, Metric spaces of non-positive curvature, Springer-Verlag, Berlin, 1999. MR 2000k:53038

[4] K. S. Brown, Buildings, Springer-Verlag, 1980. MR 90e:20001

[5] M. W. Davis, Groups generated by reflections and aspherical manifolds not covered by Euclidean space, Ann. of Math. 117 (1983), 293-324. MR 86d:57025

[6] _ Nonpositive curvature and reflection groups, in Handbook of geometric topology (Edited by R. J. Daverman and R. B. Sher), pp. 373-422, North-Holland, Amsterdam, 2002. MR 2002m:53061

[7] (1998), 297-314. MR 99b:20067

[8] E. Ghys and P. de la Harpe (ed), Sur les Groups Hyperboliques d'apres Mikhael Gromov, Progr. Math. vol. 83, Birkhäuser, Boston MA, 1990. MR 92f:53050

[9] T. Hosaka, Parabolic subgroups of finite index in Coxeter groups, J. Pure Appl. Algebra 169 (2002), 215-227. MR 2003c:20041

[10] J. E. Humphreys, Reflection groups and Coxeter groups, Cambridge University Press, 1990. MR 92h:20002

[11] G. Moussong, Hyperbolic Coxeter groups, Ph.D. thesis, The Ohio State University, 1988.

[12] J. Tits, Le problème des mots dans les groupes de Coxeter, Symposia Mathematica, vol. 1, pp. 175-185, Academic Press, London, 1969. MR 40:7339

Department of Mathematics, Utsunomiya University, Utsunomiya, 321-8505, Japan

E-mail address: hosaka@cc.utsunomiya-u.ac.jp 